\title{
Muscle insulin resistance in type 1 diabetes with coronary artery disease
}

\author{
Katherine V. Williams ${ }^{1,2}$ (D) Christina M. Shay ${ }^{1,3}$ (D) Julie C. Price ${ }^{4,5} \cdot$ Bret H. Goodpaster $^{6,7} \cdot$ Carol A. Kelley $^{6}$. \\ David E. Kelley ${ }^{6} \cdot$ Trevor J. Orchard $^{1}$ (i)
}

Received: 29 March 2020 / Accepted: 15 July 2020 / Published online: 14 September 2020

(C) Springer-Verlag GmbH Germany, part of Springer Nature 2020

\begin{abstract}
Aims/hypothesis The risk for coronary artery disease (CAD) is substantially increased in type 1 diabetes and it has been postulated that insulin resistance may contribute to this risk. The current study measured insulin resistance in type 1 diabetes with vs without $\mathrm{CAD}$ and with a focus upon skeletal muscle, to test the hypothesis that insulin resistance is more severe in participants who have type 1 diabetes and CAD. Additionally, in type 1 diabetes, we examined the hypothesis that insulin resistance is more severe in soleus (an oxidative type muscle) vs tibialis anterior (a more glycolytic type of muscle).

Methods Insulin resistance was measured in participants with type 1 diabetes with ( $n=9, \mathrm{CAD}+)$ and without CAD $(n=10$, CAD-) using euglycaemic insulin infusions combined with positron emission tomography (PET) imaging of $\left[{ }^{18} \mathrm{~F}\right]$ fluorodeoxyglucose (FDG) uptake into soleus and tibialis anterior skeletal muscles. Coronary artery calcium (CAC) score was quantified by electron beam tomography.

Results CAD+ participants with type 1 diabetes had a $>100$-fold higher CAC score than did CAD- participants with type 1 diabetes but groups did not differ in $\mathrm{HbA}_{1 \mathrm{c}}$ or insulin dose. During clamp studies, $\mathrm{CAD}+$ and $\mathrm{CAD}-$ groups had similar glucose disposal but were insulin resistant compared with historical non-diabetic participants $(n=13)$. FDG uptake by soleus muscle was similarly reduced, overall, in individuals with type 1 diabetes with or without CAD compared with non-diabetic individuals. However, FDG uptake by tibialis anterior muscle was not reduced in CAD- participants with type 1 diabetes while in CAD+ participants with type 1 diabetes it was $75 \%$ greater $(p<0.01)$. Across all participants with type 1 diabetes, FDG uptake by tibialis anterior muscle correlated positively with CAC severity.

Conclusions/interpretation Our study confirms that systemic and skeletal muscle-specific insulin resistance is seen in type 1 diabetes but found that it does not appear to be more severe in the presence of CAD. There were, however, sharp differences between soleus and tibialis anterior muscles in type 1 diabetes: while insulin resistance was clearly manifest in soleus muscle, and was of equal severity in $\mathrm{CAD}+$ and $\mathrm{CAD}-$ participants, tibialis anterior did not suggest insulin resistance in participants with type 1 diabetes, as FDG uptake by tibialis anterior correlated positively with CAC severity and was significantly increased in participants with type 1 diabetes and clinical CAD.
\end{abstract}

Electronic supplementary material The online version of this article (https://doi.org/10.1007/s00125-020-05270-w) contains peer-reviewed but unedited supplementary material, which is available to authorised users.

Katherine V. Williams

KVW3@pitt.edu

1 Department of Epidemiology, University of Pittsburgh Graduate School of Public Health, University of Pittsburgh School of Medicine, Pittsburgh, PA, USA

2 Present address: Department of Family Medicine, University of Pittsburgh School of Medicine, University of Pittsburgh, Pittsburgh, PA, USA

3 Present address: Impact and Health Metrics, American Heart Association, Dallas, TX, USA
4 Department of Radiology, University of Pittsburgh, Pittsburgh, PA, USA

5 Present address: Department of Radiology, Massachusetts General Hospital, Charlestown, MA, USA

6 Department of Medicine, Division of Endocrinology and Metabolism, Pittsburgh, PA, USA

7 Present address: Advent Health Translational Research Institute for Metabolism and Diabetes, Orlando, FL, USA 


\section{Research in context}

\section{What is already known about this subject?}

- Coronary artery disease (CAD) is increased in type 1 diabetes

- Insulin resistance is present in type 1 diabetes and epidemiological studies suggest that insulin resistance is a CAD risk factor in people with type 1 diabetes

\section{What is the key question?}

- Is systemic and skeletal muscle insulin resistance in type 1 diabetes more severe in those with vs without CAD and, as a corollary hypothesis, is skeletal muscle insulin resistance in type 1 diabetes more severe in soleus vs tibialis anterior muscle?

What are the new findings?

- In type 1 diabetes, insulin resistance (systemic and skeletal muscle-specific) is not more severe in participants with vs without $C A D$

- Soleus muscle insulin resistance was present in equal severity in type 1 diabetic participants with vs without CAD; tibialis anterior muscle insulin resistance was not present. In type 1 diabetes with CAD, glucose uptake was increased vs those without $C A D$ and vs a non-diabetic control group

- Across the cohort with type 1 diabetes, tibialis anterior glucose uptake correlated with the severity of coronary artery calcification, a marker of $C A D$

How might this impact on clinical practice in the foreseeable future?

- These findings portray a previously unrecognised complexity and diversity in the relationship between CAD and skeletal muscle metabolism in type 1 diabetes and may influence the approach to insulin resistance as a therapeutic target

Keywords Coronary artery disease $\cdot$ Insulin resistance $\cdot$ Skeletal muscle $\cdot$ Type 1 diabetes

$\begin{array}{ll}\text { Abbreviations } \\ \text { CAC } & \text { Coronary artery calcification } \\ \text { CACTI } & \text { Coronary Artery Calcification in Type 1 Diabetes } \\ \text { CAD } & \text { Coronary artery disease } \\ \text { EDC } & \text { Epidemiology of Diabetes Complications } \\ \text { FDG } & {\left[{ }^{18} \text { F }\right] \text { fluorodeoxyglucose }} \\ \text { GDR } & \text { Glucose disposal rate } \\ \text { FFM } & \text { Fat free mass } \\ \text { PET } & \text { Positron emission tomography } \\ \text { SUV } & \text { Standardised uptake value }\end{array}$

\section{Introduction}

Coronary artery disease (CAD) is the leading cause of death in individuals with type 1 diabetes with an earlier onset and higher prevalence than in those without diabetes [1, 2]. The reasons for increased CAD risk in type 1 diabetes are not fully understood. Traditional CAD risk factors of age, smoking, increased LDL-cholesterol levels and hypertension contribute to $\mathrm{CAD}$ risk in type 1 diabetes, as does poor glycaemic control, diabetes duration and end-organ renal complications [3-5]. Several epidemiological studies indicate that insulin resistance might be an additional metabolic risk factor for
CAD in type 1 diabetes [6-8], generating a hypothesis that insulin resistance could be more severe in type 1 diabetes complicated by CAD, a hypothesis tested in the current study. This postulate has pragmatic implications in considering therapies adjunctive to insulin that might mitigate insulin resistance and lessen risk for CAD.

Clinical studies employing the insulin infusion glucose clamp method, the gold standard for measuring insulin action, have found insulin resistance of moderate severity in type 1 diabetes, even in those who are non-obese [9-12], and that insulin resistance involves liver, adipose tissues and skeletal muscle [11, 13]. However, the hypothesis that insulin resistance is a CAD risk factor in type 1 diabetes emerged based on investigations that employed an estimation of insulin resistance [6-8]. An exception was the Coronary Artery Calcification in Type 1 Diabetes (CACTI) study that measured insulin resistance using a clamp and observed correlation between insulin resistance and coronary artery calcification (CAC), a strong biomarker for CAD (12). That conclusion carries an important caveat because the primary analysis included a large number of non-diabetic participants, without insulin resistance and with low CAC scores [12]. In analysis confined to participants with type 1 diabetes, the correlation between insulin resistance and CAC was no longer significant, 
leaving uncertainty as to whether insulin resistance might be more severe in those manifesting CAD in type 1 diabetes.

The goal of the current study was to test this hypothesis of more severe insulin resistance in individuals with type 1 diabetes manifesting CAD than in those who do not manifest CAD. Focus was given to measuring insulin action in skeletal muscle. Individuals with type 1 diabetes with CAD (CAD+) vs those without (CAD-), categorisations based on quantitative CAC determination, had insulin resistance measured by the glucose clamp method combined with positron emission tomography (PET) imaging of skeletal muscle uptake of the glucose analogue $\left[{ }^{18} \mathrm{~F}\right]$ fluorodeoxyglucose (FDG). FDG uptake was measured separately in soleus and tibialis anterior muscles of the leg. Based on PET imaging, our group has reported that soleus manifests higher insulin sensitivity in healthy, lean non-diabetic individuals than does tibialis anterior [14-16]. These muscles differ in fibre-type composition [17]: soleus is comprised predominantly of type 1 , slowtwitch oxidative muscle fibres; and tibialis anterior has a higher proportion of type 2, fast-twitch, glycolytically oriented fibres. A large body of animal data indicates a role for muscle fibre type in insulin sensitivity, heightened in oxidative muscle [18-20]. Enabled by PET imaging, which provides a spatial mapping of metabolism, our objective in the current study was to test the hypothesis that differences in muscle insulin resistance are evident in type 1 diabetes and that insulin resistance is more severe in the oxidative soleus vs the more glycolytic tibialis anterior. The findings that address this issue additionally frame a provocative new line of evidence linking CAD and skeletal muscle metabolism in type 1 diabetes.

\section{Methods}

Study population Participants with childhood onset $(<17$ years old) type 1 diabetes were recruited from the 493 Pittsburgh Epidemiology of Diabetes Complications (EDC) study [21, 22 ] at the 18 year examination. CAC was assessed via electron beam tomography (EBT) using a GE-Imatron ultrafast computed tomography scanner (GEImatron, San Francisco, CA, USA), and CAC scores [23] and area were calculated [24]. CAD was defined based on a history of confirmed atherosclerotic cardiovascular clinical event, myocardial infarction confirmed by $\mathrm{Q}$ waves on an ECG (Minnesota codes 1.1 or 1.2), or hospital records, angiographic stenosis $\geq 50 \%$, revascularisation, EDC physician-diagnosed angina, or ischaemic ECG changes. Absence of CAD was defined as no clinical history of CAD and a CAC score $<200$ Agatston units. Healthy non-diabetic historical control individuals (status confirmed by fasting glucose measures, $\mathrm{HbA}_{1 \mathrm{c}}$ and medical examination) were recruited by advertisement. The University of Pittsburgh Institutional Review Board approved this study.
Written informed consent was obtained prior to participation. Assessment of additional clinical variables (height, weight, glucose, cholesterol, HbAlc and renal measures) and details of all procedures (exclusion criteria and assessment of CAC and fat free mass [FFM]) are described in electronic supplemental materials (ESM) Methods.

Glucose clamp procedure and PET imaging of skeletal muscle FDG uptake During the euglycaemic clamp procedure to assess insulin sensitivity/resistance, an insulin infusion rate of $40 \mathrm{mU} \mathrm{m}^{-2} \mathrm{~min}^{-1}$ (Humulin; Eli Lilly, Indianapolis, IN, USA) was used (see ESM Methods for details). PET imaging was initiated after at least $60 \mathrm{~min}$ of the clamp and once steady-state conditions were achieved. PET imaging was performed using a Siemens/CTI ECAT HR+ tomograph (USA) in three-dimensional imaging mode (63 parallel plans; axial field-of-view $15.2 \mathrm{~cm}$, slice width $2.4 \mathrm{~mm}$ ) as previously described [25].

For seven of the nine CAD+ participants with type 1 diabetes, radial artery catheterisation was not performed due to concerns that any ensuing arterial injury could compromise later use of the vessel if coronary revascularisation were needed. Instead, in these individuals, glucose values during the clamp were measured using sampling obtained from a dorsal hand vein warmed by the heated hand technique and an alternative approach to the determination of FDG uptake into muscle was employed (see ESM Methods for details).

MRI was performed using a General Electric 3.0 Tesla Signa MRI scanner (USA), including a body coil and images were co-registered with PET images to enhance anatomic accuracy in placing regions of interest (ROI) on soleus and tibialis anterior muscles (see ESM Methods and ESM Fig. 1 for details).

Skeletal muscle FDG time-activity curves and the arterial input function data were applied in a standard Patlak graphical analysis to determine the influx constant $\left(\mathrm{K}\left[\mathrm{ml} \mathrm{min}^{-1} \mathrm{~g}\right]\right)$ in muscle [26] over the final 15-60 min. For participants deemed ineligible for arterial sampling due to their underlying CAD+ disease status, a standardised uptake value (SUV) ratio was used to determine Patlak K (see ESM Methods and ESM Fig. 2 for details).

Statistical analysis Data were summarised using descriptive statistics and distributions of continuous variables were assessed for normality. Non-parametric statistics (Wilcoxon rank sum test, Kruskal-Wallis, Mann-Whitney and Spearman's $\rho$ ) were used if variables were not normally distributed (FDG uptake, CAC measures). Logistic regression and multiple linear regression were used to adjust for categorical and continuous variables, respectively. For categorical variables, $\chi^{2}$ analyses were used to compare frequencies; Fisher's Exact test was performed if expected counts were $<5$. A $p$ value of $<0.05$ was considered statistically significant. 
All statistical analyses were performed using SPSS version 24 (IBM Corp, Armonk, NY, USA).

\section{Results}

Characteristics of participants Clinical characteristics of participants are shown in Table 1. Men and women were equally represented in the type 1 diabetes $\mathrm{CAD}^{-}$and CAD+ groups. Mean CAC score was two orders of magnitude higher in CAD+ compared with CAD- participants with type 1 diabetes (considerably above the clinically accepted threshold of 400 Agatston units used to designate presence of CAD); in the CAD- group, CAC scores were in the range of $0-48$ Agatston units. Participants with type 1 diabetes in the CADgroup had a mean age of $43.1 \pm 5.7$ years and their diabetes was long-standing (mean duration $35.0 \pm 6.0$ years). Those in the $\mathrm{CAD}+$ group were approximately a decade older and had nearly a decade longer duration of type 1 diabetes. Regarding other $\mathrm{CAD}$ risk factors, the two groups did not differ in $\mathrm{HbA}_{1 \mathrm{c}}$, insulin dose or lipid variables, and there was no difference in creatinine levels or eGFR (estimated by the chronic kidney disease epidemiology [CKD-EPI] equation). The type 1 diabetes CAD+ group of participants had a higher mean BMI, a higher waist circumference (although not statistically significant $[p=0.07$ controlled for sex $]$ ) and higher systolic BP. The control group of 13 non-diabetic individuals had similar mean age and $\mathrm{BMI}$ to $\mathrm{CAD}-$ participants with type 1 diabetes, but were younger and leaner than $\mathrm{CAD}+$ participants with type 1 diabetes.

Insulin-stimulated systemic glucose disposal During the insulin infusion of $40 \mathrm{mU} \mathrm{m}{ }^{-2} \mathrm{~min}^{-1}$, stable euglycaemia was maintained in non-diabetic participants, and CAD- and $\mathrm{CAD}+$ participants with type 1 diabetes. Steady-state glucose disposal rate (GDR) during the clamp is shown in Fig. 1. CADand $\mathrm{CAD}+$ participants with type 1 diabetes were insulin resistant compared with non-diabetic participants, with an approximately $30 \%$ mean reduction in GDR $(p<0.05)$. Insulin resistance was of similar severity in $\mathrm{CAD}+$ and $\mathrm{CAD}^{-}$participants with type 1 diabetes. An effect of sex on severity of insulin resistance in type 1 diabetes was not observed.
Table 1 Characteristics of participants

\begin{tabular}{|c|c|c|c|}
\hline \multirow[t]{2}{*}{ Characteristic } & \multirow[t]{2}{*}{ No diabetes (control group) } & \multicolumn{2}{|c|}{ Type 1 diabetes } \\
\hline & & No CAD & With CAD \\
\hline$n$ (male/female) & $13(6 / 7)$ & $10(5 / 5)$ & $9(5 / 4)$ \\
\hline Age, years & $40.4 \pm 9.7$ & $43.1 \pm 5.7$ & $52.4 \pm 7.1 * *$ \\
\hline Diabetes duration, years & - & $35.0 \pm 6.0$ & $42.2 \pm 7.7^{*}$ \\
\hline Weight, kg & $72.7 \pm 13.6$ & $70.4 \pm 13.0$ & $77.3 \pm 15.0$ \\
\hline BMI, $\mathrm{kg} / \mathrm{m}^{2}$ & $24.1 \pm 2.4$ & $24.3 \pm 3.7$ & $28.0 \pm 4.0 *$ \\
\hline Waist circumference, $\mathrm{cm}$ & - & $85.2 \pm 13.3$ & $91.7 \pm 9.6$ \\
\hline Systolic BP, mmHg & - & $116 \pm 17$ & $137 \pm 23^{*}$ \\
\hline Diastolic BP, mmHg & - & $69 \pm 11$ & $71 \pm 9$ \\
\hline Insulin dose, $\mathrm{U} / \mathrm{kg}$ & - & $0.59 \pm 0.29$ & $0.59 \pm 0.20$ \\
\hline $\mathrm{HbA}_{1 \mathrm{c}}, \mathrm{mmol} / \mathrm{mol}$ & $34 \pm 2$ & $60 \pm 10 * * *$ & $60 \pm 8 * * *$ \\
\hline $\mathrm{HbA}_{1 \mathrm{c}}, \%$ & $5.3 \pm 0.29$ & $7.6 \pm 1.3 * * *$ & $7.6 \pm 1.0 * * *$ \\
\hline LDL-cholesterol, mmol/l & $2.90 \pm 0.41$ & $2.33 \pm 0.75$ & $2.33 \pm 0.70$ \\
\hline Triacylglycerols, mmol/l & $0.98 \pm 0.32$ & $0.82 \pm 0.38$ & $0.97 \pm 0.42$ \\
\hline HDL-cholesterol, mmol/1 & $1.63 \pm 0.34$ & $1.42 \pm 0.36$ & $1.45 \pm 0.44$ \\
\hline Creatinine, $\mu \mathrm{mol} / 1$ & - & $68.63 \pm 7.63$ & $76.25 \pm 15.25$ \\
\hline 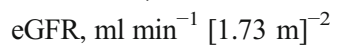 & - & $93.1 \pm 16.2$ & $78.9 \pm 20.1$ \\
\hline \multicolumn{4}{|l|}{ CAC scores } \\
\hline Agatston units & - & $6.5 \pm 15.3$ & $969.0 \pm 848.5^{* *}$ \\
\hline Area & - & $2.5 \pm 5.5$ & $264.2 \pm 222.0 * *$ \\
\hline Volume & - & $7.6 \pm 16.5$ & $792.5 \pm 665.9 * *$ \\
\hline Density $^{a}$ & - & $2.2 \pm 1.2$ & $3.5 \pm 0.5$ \\
\hline
\end{tabular}

Data are means $\pm \mathrm{SD}$

${ }^{\text {a }}$ CAC density $(n=11)$ could not be calculated in those with Agatston values of zero

$* p<0.05, * * p<0.01$ and $* * * p<0.001$ for type 1 diabetes vs no diabetes $\left(\mathrm{HbA}_{1 \mathrm{c}}\right)$ and for with vs without $\mathrm{CAD}$ for the remaining variables 


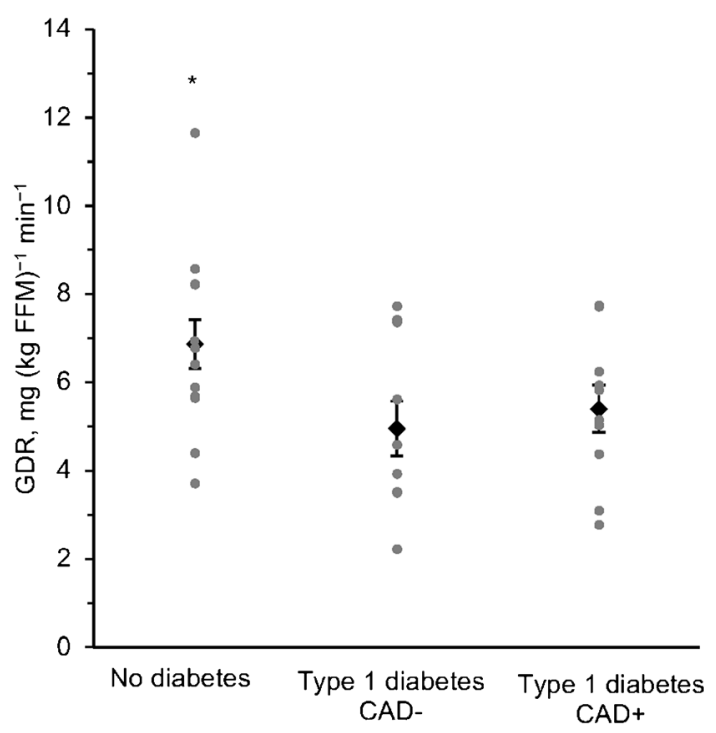

Fig. 1 Insulin-stimulated systemic GDR in non-diabetic individuals and individuals with type 1 diabetes with and without CAD, and type 1 diabetes without and with CAD determined during insulin infusion euglycaemic clamp studies. Systemic glucose disposal was greater in non-diabetic individuals than in those with type 1 diabetes but rates did not differ significantly between the two type 1 diabetes groups (with vs without $\mathrm{CAD}$ ). Individual data points are shown and summarised as means \pm SE. $* p<0.05$ Mann-Whitney test, no diabetes vs type 1 diabetes, with or without $\mathrm{CAD}$

PET imaging of skeletal muscle insulin-stimulated FDG uptake FDG uptake in soleus muscle was approximately $40 \%$ lower in participants with type 1 diabetes compared with nondiabetic participants (Fig. 2), indicative of a moderate severity of skeletal muscle insulin resistance in soleus muscle in type 1 diabetes. Nearly all participants with type 1 diabetes manifested insulin resistance in soleus muscle. As with systemic GDR, we found that soleus insulin resistance was of equal severity in $\mathrm{CAD}+$ and $\mathrm{CAD}^{-}$participants with type 1 diabetes.

The data on FDG uptake by tibialis anterior muscle (Fig. 2) do however reveal differences between the CAD- and CAD+ participants with type 1 diabetes and differences from the insulin resistance observed in soleus muscle. In the type 1 diabetes $\mathrm{CAD}^{-}$group, FDG uptake by tibialis anterior muscle was not significantly different from that in the non-diabetic group, denoting an absence of insulin resistance for this muscle group in CAD- participants with type 1 diabetes, unlike the respective findings for soleus muscle. Moreover, in the type 1 diabetes CAD+ group, FDG uptake in tibialis anterior muscle was approximately $75 \%$ higher $(p<0.01)$ than in the type 1 diabetes CAD- group (and equally increased compared with values in non-diabetic participants). Thus, values for FDG uptake by tibialis anterior muscle in $\mathrm{CAD}+$ participants with type 1 diabetes revealed an unexpected finding of heightened insulin sensitivity, which was observed in nearly all the participants in the CAD+ group.

In the non-diabetic individuals, FDG uptake by soleus muscle was higher than that by tibialis anterior muscle $(p<0.01)$, consistent with our prior observations [14-16]. However, in the type 1 diabetes $\mathrm{CAD}-$ group there was no difference between these muscles in FDG uptake, a parity attributable to insulin resistance in soleus but not tibialis anterior muscle. In the type 1 diabetes CAD+ group, as noted above, FDG uptake by tibialis anterior muscle was higher than in soleus muscle $(p<0.001)$, thus reversing the gradient between these muscles that was observed in the non-diabetic individuals.

Concerning a technical issue of the denominator used to express Patlak K; as scaled for FFM (similar in type 1 diabetes without CAD $54.1 \pm 10.3 \mathrm{~kg}$ and type 1 diabetes with CAD51.4 $\pm 12.2 \mathrm{~kg}$ ), values for $\mathrm{K}$ were highly correlated with K scaled to weight $(r=0.90, p<0.001$ for soleus and $r=0.93$, $p<0.001$ for tibialis anterior). As such, type 1 diabetes group differences were consistent whether $\mathrm{K}$ was scaled for weight (Fig. 2) or FFM (data not shown).

Correlates of insulin action in type 1 diabetes Table 2 shows the correlation analyses for insulin action employing data from the participants with type 1 diabetes. Negative correlations with systemic GDR in type 1 diabetes were observed for BMI, waist circumference and triacylglycerols; these associations were also evident when analyses included non-diabetic individuals $(r=-0.49, p<0.01$ for BMI; $r=-0.37, p<0.05$ for triacylglycerols). Not unexpectedly, in type 1 diabetes, daily insulin dose normalised to weight was a negative correlate of GDR. $\mathrm{HbA}_{1 \mathrm{c}}$, LDL-cholesterol and HDL-cholesterol were not significant correlates; eGFR showed a negative association (although this did not reach statistical significance, $p=$ $0.22)$.

Regarding insulin action in skeletal muscle, FDG uptake by soleus and tibialis anterior muscles was each correlated with systemic GDR, whether including non-diabetic individuals or in analysis restricted to type 1 diabetes (the latter findings are shown in Table 2). In stepwise regression analysis using data from all participants with type 1 diabetes, FDG uptake in each muscle retained significance in relation to GDR; together, FDG uptake by the two muscles accounted for half of the variance $\left(R^{2}=0.49, p<0.001\right)$ in GDR in type 1 diabetes. In type 1 diabetes, FDG uptake by soleus muscle was negatively correlated with BMI and waist circumference, conforming to the associations noted for systemic GDR. However, these were not significant correlates for tibialis anterior FDG uptake in participants with type 1 diabetes. Rather, a striking observation was a strong positive correlation between tibialis anterior FDG uptake and severity of CAC score $(r=$ $0.61 ; p<0.01)$. For soleus muscle in participants with type 1 diabetes, the association between FDG uptake and CAC score did not reach statistical significance $(r=0.11 ; p=0.65)$. Scatterplots for FDG uptake and CAC are shown in ESM Fig. 3. The correlation between FDG uptake by tibialis anterior and $\mathrm{CAC}$ score persisted after excluding participants with 


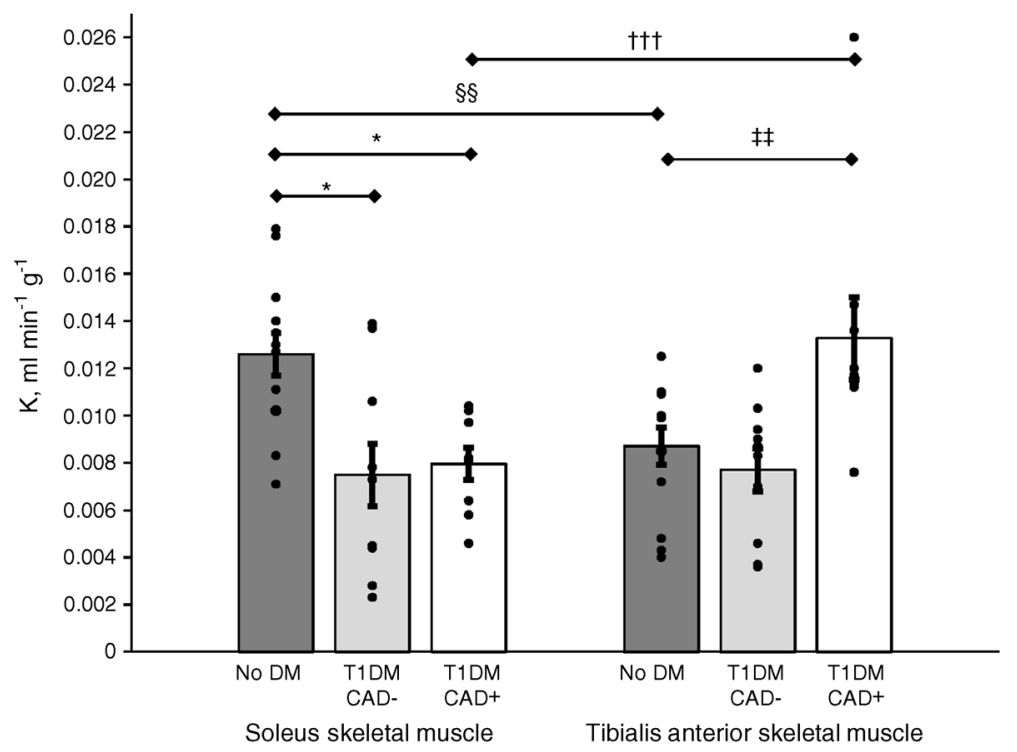

Fig. 2 Insulin-stimulated uptake of FDG $(\mathrm{K})$ in soleus and tibialis anterior skeletal muscles in participants with no diabetes (No DM; $n=13$ ), CAD - participants with type 1 diabetes (T1DM CAD-; $n=10)$ and CAD+ participants with type 1 diabetes (T1DM CAD+; $n=9$ ). Individual data points are shown and summarised as means \pm SE. Within the soleus muscle, FDG uptake was reduced in participants with type 1 diabetes with and without CAD $(p<0.05)$. Within the tibialis anterior muscle, FDG uptake was higher in CAD+ participants with type 1 diabetes $(p<0.01)$. In non-diabetic individuals, FDG uptake in tibialis anterior muscle was lower than in soleus muscle $(p<0.01)$. In CAD-

participants with type 1 diabetes, no difference in FDG uptake was observed when comparing soleus with tibialis anterior muscle. In CAD+ participants with type 1 diabetes, FDG uptake was greater in tibialis anterior muscle than in soleus muscle $(p<0.001) . * p<0.05$, Mann-Whitney test, compared with non-diabetic individuals; ${ }^{\sharp} p<0.01$, Mann-Whitney test in tibialis anterior muscle in CAD+ individuals with type 1 diabetes vs non-diabetic individuals; ${ }^{\S \S} p<0.01$, Wilcoxon rank sum test in tibialis anterior vs soleus muscle in non-diabetic individuals; ${ }^{\dagger \dagger} p<0.001$, Wilcoxon rank sum in tibialis anterior vs soleus muscle in CAD+ participants with type 1 diabetes

type 1 diabetes who had a CAC score of zero. CAC density adjusted for volume was also positively correlated with tibialis anterior FDG uptake. In participants with type 1 diabetes, age correlated with CAC score $(p<0.01)$; together, age and tibialis anterior FDG uptake were robust predictors of CAC score $\left(R^{2}=0.81\right)$. Scatterplots for age, FDG uptake and CAC are shown in ESM Fig. 4.

\section{Discussion}

Insulin resistance is considered a risk factor for CAD in the metabolic syndrome and in type 2 diabetes [27] and is postulated to contribute towards risk for CAD in type 1 diabetes [28]. CAD is the leading cause of death in type 1 diabetes; traditional risk factors and glycaemic control contribute but do

Table 2 Correlations between clinical characteristics and systemic glucose disposal and for skeletal muscle-specific FDG uptake in type 1 diabetes $(n=19)$

\begin{tabular}{|c|c|c|c|}
\hline Characteristic & Glucose disposal & Soleus K & Tibialis anterior $\mathrm{K}$ \\
\hline Age, years & 0.34 & -0.04 & 0.42 \\
\hline BMI, $\mathrm{kg} / \mathrm{m}^{2}$ & $-0.61 * *$ & $-0.52 *$ & -0.31 \\
\hline Waist circumference, $\mathrm{cm}$ & $-0.62 * *$ & $-0.46^{*}$ & -0.19 \\
\hline Triacylglycerol, mmol/1 & $-0.68 * *$ & -0.38 & -0.37 \\
\hline LDL-cholesterol, mmol/1 & -0.23 & -0.24 & -0.02 \\
\hline HDL-cholesterol, mmol/1 & 0.22 & -0.19 & -0.24 \\
\hline $\mathrm{HbA}_{1 \mathrm{c}}, \mathrm{mmol} / \mathrm{mol}$ & 0.31 & 0.06 & 0.38 \\
\hline Insulin dose, $\mathrm{U} / \mathrm{kg}$ & $-0.60 * *$ & -0.34 & -0.14 \\
\hline CAC score & 0.41 & 0.11 & $0.61 * *$ \\
\hline CAC volume & 0.41 & 0.06 & $0.56^{*}$ \\
\hline CAC density ${ }^{\mathrm{a}}$ & 0.28 & 0.26 & 0.65 \\
\hline GDR, mg (kg FFM) $)^{-1} \min ^{-1}$ & - & $0.62 * *$ & $0.56^{* *}$ \\
\hline
\end{tabular}

${ }^{\mathrm{a}} \mathrm{CAC}$ density was corrected for CAC volume; CAC density $(n=11)$ could not be calculated in those with values for CAC volume of zero

${ }^{*} p<0.05$ and $* * p<0.01$ 
not fully explain the increased risk of CAD in type 1 diabetes. Autoimmune destruction of pancreatic beta cells is central in the pathogenesis of type 1 diabetes, yet insulin resistance is also commonly present [11-13]. Clinical studies that initially described insulin resistance in type 1 diabetes several decades ago involved individuals with poor glycaemic control and ascribed insulin resistance to 'glucotoxicity' $[9,10]$. More contemporary studies, involving participants with type 1 diabetes under good glycaemic control, who were nonsedentary and non-obese, consistently observe insulin resistance $[11,12]$. The current study was undertaken to test the hypothesis that insulin resistance is more severe in type 1 diabetes complicated by CAD than in type 1 diabetes where $\mathrm{CAD}$ is absent and, as a corollary, to examine this issue in skeletal muscle. Moreover, we explored whether there is heterogeneity of insulin resistance severity in different muscle groups in type 1 diabetes and whether heterogeneity of muscle insulin resistance may be associated with CAD. Our working hypothesis was that in type 1 diabetes, insulin resistance would be more severe in soleus (an oxidative muscle) than in tibialis anterior (a more glycolytically oriented muscle).

Prior analyses from our group, using an estimation of insulin resistance, suggested that insulin resistance augments risk for CAD in type 1 diabetes [6], a postulate later supported by other epidemiological studies that also estimated rather than directly measured insulin resistance $[7,8]$. The CACTI study was important in addressing this question because it both measured insulin resistance by the glucose clamp method and ascertained CAC scores in a cohort of 40 individuals with type 1 diabetes and 47 non-diabetic individuals [12]. Across all participants, a significant correlation between severity of insulin resistance and CAC score was found. Yet, in analyses restricted to the 40 participants with type 1 diabetes, the correlation between $\mathrm{CAC}$ and insulin resistance was not statistically significant [12].

In the current study, we confirmed the presence of insulin resistance in type 1 diabetes, yet we found that insulin resistance was equally severe in participants with vs without CAD. Therefore, our findings are consistent with the CACTI findings [12]. The lack of a significant association between severity of insulin resistance and CAC score reported for participants with type 1 diabetes does not disprove that insulin resistance may contribute risk for CAD. In type 1 diabetes, insulin resistance may constitute a 'common soil' interacting with other CAD risk factors, which is how insulin resistance is construed to contribute towards CAD risk in the metabolic syndrome and type 2 diabetes. Intervention-based studies may prove more telling than cross-sectional analyses in testing the impact of insulin resistance upon risk for CAD in type 1 diabetes.

Under the metabolic conditions of a euglycaemic clamp, skeletal muscle accounts for most of the glucose utilisation and is a primary locus of insulin resistance. In the current study, PET imaging of FDG uptake by skeletal muscle was performed to assess insulin resistance in type 1 diabetes. PET images were co-registered with MRI of the calf, which enabled FDG uptake to be assessed separately in soleus and tibialis anterior muscles and novel data emerged concerning associations between insulin resistance in type 1 diabetes and CAD. In type 1 diabetes, insulin resistance was observed in soleus muscle and was of equal severity in participants with vs without $\mathrm{CAD}$, further rejecting the hypothesis that insulin resistance is more severe in type 1 diabetes plus CAD. We did not, however, observe insulin resistance in tibialis anterior muscle in participants with type 1 diabetes; this supports our secondary hypotheses of heterogeneity between muscles in insulin resistance and that more oxidative muscle manifests greater insulin resistance than a more glycolytically oriented muscle.

In the participants with type 1 diabetes who were CAD-, the FDG uptake by tibialis anterior muscle was equal to that observed in non-diabetic individuals, denoting an absence of insulin resistance. Furthermore, in the participants with type 1 diabetes who were CAD+, FDG uptake by tibialis anterior muscle was approximately $75 \%$ higher than in either nondiabetic individuals or $\mathrm{CAD}-$ participants with type 1 diabetes. Most striking, we observed that across all participants with type 1 diabetes, FDG uptake by tibialis anterior muscle was significantly correlated with severity of CAC score. Age and FDG uptake by tibialis anterior together accounted for $81 \%$ of the variance in CAC, a marked combined effect. These two observations, elevated FDG uptake by tibialis anterior muscle in the presence of CAD and correlation of FDG uptake by tibialis anterior with CAC scores, are indicative of an association between $\mathrm{CAD}$ and skeletal muscle metabolism in type 1 diabetes.

Our finding of insulin resistance in soleus muscle in the 19 adult participants with type 1 diabetes is not novel and is highly consistent with several recent reports. Cree-Green et al [29] used magnetic resonance spectroscopy of calf muscle to measure skeletal muscle bioenergetics in 17 adolescents with type 1 diabetes. Surface coils were placed to focus on soleus muscle and slower rates of post-exercise ATP recovery in type 1 diabetes were found, correlating with diminished insulin-stimulated glucose disposal. Kacerovsky et al [30], when comparing eight non-obese adults with type 1 diabetes vs a non-diabetic control group, found that insulin-stimulated ATP production and the accumulation of glucose 6-phosphate (as an index of glucose transport) in soleus muscle were diminished in type 1 diabetes. Peltoniemi et al [31] used PET imaging to measure insulin-stimulated FDG uptake in thigh muscles (of mixed fibre-type composition) in eight non-obese young adults with type 1 diabetes and observed a decrease of nearly $50 \%$. In a PET imaging study of FDG uptake in 15 men with CAD conducted during a glucose clamp, Paternostro et al [32] observed insulin resistance in 
skeletal muscles of the arm and cardiac muscle compared with control individuals. Additional PET studies have found that FDG uptake in the heart in type 1 diabetes compared with controls is no different despite lower FDG uptake in the leg [33], thus, cardiac muscle may not manifest insulin resistance in the face of systemic insulin resistance.

In the current study, insulin resistance was not found in tibialis anterior muscle in participants with type 1 diabetes. Indeed, FDG uptake by tibialis anterior was approximately $75 \%$ higher in $\mathrm{CAD}+$ participants with type 1 diabetes than in either non-diabetic individuals or the diabetic CAD- participants. The direct contribution of tibialis anterior muscle to systemic glucose disposal can only be minor given its small size, yet there was strong correlation between FDG uptake by this muscle and systemic glucose disposal in type 1 diabetes. This suggests that FDG uptake in tibialis anterior muscle is properly representative of a meaningfully larger proportion of overall skeletal muscle mass, a rationale also applicable to soleus muscle, itself representing a small fraction of muscle mass yet its FDG uptake correlated with systemic glucose disposal. PET imaging of FDG uptake in skeletal muscle yields high fidelity measures with strong correlation with systemic glucose disposal [14, 25, 33, 34]. Thus, our interpretation is that FDG uptake by soleus and tibialis anterior muscles, though differing regarding the presence vs absence of insulin resistance, respectively, are each likely representative of patterns of glucose utilisation by larger proportions of muscle mass in type 1 diabetes. This interpretation is supported by independent significant contributions of each muscle to GDR in multiple regression analysis.

Quantitative in vivo human data on metabolic diversity among different skeletal muscles are sparse yet the phenomenon is well described in animal studies [18-20]. In animal studies, muscles comprised of type 1 fibres display higher insulin sensitivity than muscle comprised of type 2 fibres. This is attributed to metabolic differences, collectively characterised as being more oxidative in type 1 and more glycolytic in type 2 fibres. Metabolic properties associated with fibre type include differences in pathways considered to shape insulin action and glucose and lipid metabolism [35-37]. In humans, soleus muscle is comprised predominantly of type 1 fibres whereas tibialis anterior muscle has a more substantial contribution of type 2 fibres [38, 39]. Our data on insulin-stimulated FDG uptake by soleus and tibialis anterior muscles in healthy volunteers, reported herein and previously $[14,15]$, reveal higher insulin sensitivity in oxidative muscle. Turning to pathophysiology, there are data suggesting that insulin resistance in type 2 diabetes and obesity may predominantly involve type 1 fibres $[36,40]$. Thus, metabolic characteristics that in health dispose towards greater insulin sensitivity may in metabolic diseases create vulnerability to insulin resistance. The loci of this vulnerability to insulin resistance in type 1 fibres centre on mitochondrial and lipid metabolism.
However, mitigation of the insulin resistance in tibialis anterior (relative to soleus) muscle that was observed in type 1 diabetes are data that to our knowledge are largely without precedent in either human or animal studies. One precedent is the study by $\mathrm{Ng}$ et al [14], in which among seven obese insulin-resistant non-diabetic participants, insulin resistance was significantly evident in soleus muscle yet only nominally so in tibialis anterior muscle.

Most notably, in the current study, the strong positive correlation among participants with type 1 diabetes (both $\mathrm{CAD}+$ and $\mathrm{CAD}^{-}$) between $\mathrm{CAC}$ score and FDG uptake by tibialis anterior muscle is provocative. The correlation was not evident for soleus muscle despite its insulin resistance. The underlying mechanism(s) that link the amount of detectable atherosclerotic plaque in the coronary arteries (for which CAC score is a robust biomarker) in type 1 diabetes and insulinstimulated glucose utilisation in tibialis anterior are not known. Further research is needed to understand the association, whether it is restricted to type 1 diabetes or exists more broadly in CAD regardless of diabetes status and how might skeletal muscle metabolism interact with effects of ageing in relation to CAD? Which other skeletal muscles might associate like tibialis anterior with $\mathrm{CAD}$, and are these too characterised by a disposition towards glycolytic metabolism?

The current study has several limitations. Though both groups of participants with type 1 diabetes (CAD+ and $\mathrm{CAD}-$ ) used similar daily doses of insulin (weight normalised), and had similar $\mathrm{HbA}_{1 c}$, those participants who were $\mathrm{CAD}+$ were approximately a decade older, had a longer duration of diabetes, a higher $\mathrm{BMI}$ and higher BP than those who were CAD-. These differences would presumably dispose the $\mathrm{CAD}+$ diabetic individuals to more severe insulin resistance, but differences in insulin resistance were not observed between those with vs without CAD. A methodological limitation is that seven of the CAD+ participants with type 1 diabetes did not have arterial cannulation for the PET studies, due to concerns for potential injury of the vessel in the event of a future medical need for harvesting a radial artery for coronary artery surgery. Thus, these participants did not have arterial FDG time-activity data. This was addressed by deriving muscle-specific linear regression equations to relate $\mathrm{K}$ determined by standard Patlak analysis in those with arterial lines $(n=12)$ to SUVs for FDG uptake. K determined by Patlak and SUV data were highly correlated. A third limitation is the use of historical data for the non-diabetic participants, data for whom have previously been reported [14, 25]. PET imaging studies performed in conjunction with glucose clamps are complex, invasive and costly, and for the current study data for nondiabetic individuals were used chiefly to frame comparisons between the absence and presence of CAD in type 1 diabetes.

In summary, our data confirm insulin resistance in type 1 diabetes but do not support a postulate that insulin resistance is either selective for or more severe in those with CAD than without $\mathrm{CAD}$. Insulin resistance was evident in soleus muscle 
in type 1 diabetes, consistent with recent reports and soleus insulin resistance was equal in $\mathrm{CAD}+$ and $\mathrm{CAD}-$ participants with type 1 diabetes. However, in type 1 diabetes, FDG uptake by tibialis anterior muscle was not resistant to insulin. In CAD+ participants with type 1 diabetes, this uptake was significantly increased and was correlated with CAC scores, the mechanisms for which remain to be elucidated. These findings portray a previously unrecognised complexity and diversity in insulin-stimulated skeletal muscle metabolism in type 1 diabetes and point to an association between $\mathrm{CAD}$ and the metabolism of some skeletal muscles.

Data availability Data are available on request from the authors.

Funding This work was supported by National Institutes of Health (NIH), National Institute of Diabetes and Digestive and Kidney Diseases (NIDDK) DK071487 and DK034818 grants.

Authors' relationships and activities The authors declare that there are no relationships or activities that might bias, or be perceived to bias, their work.

Contribution statement TJO, DEK and JCP designed the study. CS, CAK, BHG, DEK and JCP contributed to participant recruitment, clamp and PET studies and initial compilation of data. KVW completed data analysis and wrote the manuscript with input from DEK, JCP, BHG and TJO. All co-authors reviewed the manuscript and give final approval of the version to be published. TJO serves as guarantor for the study design, data collection and access, and the decision to publish the manuscript.

\section{References}

1. De Ferranti SD, De Boer IH, Fonseca V et al (2014) Type 1 diabetes mellitus and cardiovascular disease: a scientific statement from the American Heart Association and American Diabetes Association. Diabetes Care 37:2843-2863. https://doi.org/10.2337/dc14-1720

2. Miller RG, Mahajan HD, Costacou T, Sekikawa A, Anderson SJ, Orchard TJ (2016) A contemporary estimate of total mortality and cardiovascular disease risk in young adults with type 1 diabetes: the Pittsburgh Epidemiology of Diabetes Complications Study. Diabetes Care 1:1-8. https://doi.org/10.2337/dc16-1162

3. Zgibor JC, Piatt GA, Ruppert K, Orchard TJ, Roberts MS (2006) Deficiencies of cardiovascular risk prediction models for type 1 diabetes. Diabetes Care 29(8):1860-1865. https://doi.org/10.2337/ dc06-0290

4. Zgibor J, Ruppert K, Orchard T et al (2010) Development of a coronary heart disease risk prediction model for type 1 diabetes: the Pittsburgh CHD in type 1 diabetes risk model. Diabetes Res Clin Pract 88(3):314-321 8p. https://doi.org/10.1016/j.diabres. 2010.02.009

5. Nathan DM, Bebu I, Braffett BH et al (2016) Risk factors for cardiovascular disease in type 1 diabetes. Diabetes 65(5):13701379. https://doi.org/10.2337/db15-1517

6. Orchard T, Olson J, Erbey J, Williams K (2003) Insulin resistancerelated factors, but not glycemia, predict coronary artery disease in type 1 diabetes. Diabetes 26(5):1374-1379

7. Kilpatrick ES, Rigby AS, Atkin SL (2007) Insulin resistance, the metabolic syndrome, and complication risk in type 1 diabetes: 'double diabetes in the diabetes control and complications trial. Diabetes Care 30(3):707-712. https://doi.org/10.2337/dc06-1982
8. Bjornstad P, Maahs DM, Duca LM et al (2016) Estimated insulin sensitivity predicts incident micro- and macrovascular complications in adults with type 1 diabetes over 6 years: the coronary artery calcification in type 1 diabetes study. J Diabetes Complicat 30(4): 586-590. https://doi.org/10.1016/j.jdiacomp.2016.02.011

9. DeFronzo RA, Hendler R, Simonson D (1982) Insulin resistance is a prominent feature of insulin-dependent diabetes. Diabetes 31(9): 795-801. https://doi.org/10.2337/diab.31.9.795

10. Yki-Järvinen H, Koivisto VA (1984) Insulin sensitivity in newly diagnosed type 1 diabetics after ketoacidosis and after three months of insulin therapy. J Clin Endocrinol Metab 59(3):371-378. https:// doi.org/10.1210/jcem-59-3-371

11. Bergman BC, Howard D, Schauer IE et al (2012) Features of hepatic and skeletal muscle insulin resistance unique to type 1 diabetes. $\mathrm{J}$ Clin Endocrinol Metab 97(5):1663-1672. https://doi.org/10.1210/ jc.2011-3172

12. Schauer IE, Snell-Bergeon JK, Bergman BC et al (2011) Insulin resistance, defective insulin-mediated fatty acid suppression, and coronary artery calcification in subjects with and without type 1 diabetes: the CACTI study 4460. Diabetes 60(1):306-314

13. Cree-Green M, Stuppy JJ, Thurston J et al (2018) Youth with type 1 diabetes have adipose, hepatic, and peripheral insulin resistance. J Clin Endocrinol Metab 103(10):3647-3657. https://doi.org/10. 1210/jc.2018-00433

14. Ng JM, Bertoldo A, Minhas DS et al (2014) Dynamic PET imaging reveals heterogeneity of skeletal muscle insulin resistance. J Clin Endocrinol Metab 99(1):E102-E106. https://doi.org/10.1210/jc. 2013-2095

15. Bertoldo A, Pencek RR, Azuma K et al (2006) Interactions between delivery, transport, and phosphorylation of glucose in governing uptake into human skeletal muscle. Diabetes 55(11):3028-3037. https://doi.org/10.2337/db06-0762

16. Pencek RR, Bertoldo A, Price J, Kelley C, Cobelli C, Kelley DE (2006) Dose-responsive insulin regulation of glucose transport in human skeletal muscle. Am J Physiol Endocrinol Metab 290(6): E1124-E1130. https://doi.org/10.1152/ajpendo.00598.2004

17. Yang JC, Yoo JY (1986) Histochemical muscle fiber types of autopsied human gastrocnemius, soleus, peroneus longus and tibialis anterior muscles. J Pathol Transl Med 20(4):413-426

18. Garetto LP, Richter EA, Goodman MN, Ruderman NB (1984) Enhanced muscle glucose metabolism after exercise in the rat: the two phases. Am J Physiol Metab 266(6 pt 1):E471-E475. https:// doi.org/10.1152/ajpendo.1984.246.6.e471

19. Bonen A, Tan MH, Watson-Wright WM (1981) Insulin binding and glucose uptake differences in rodent skeletal muscles. Diabetes 30(8):702-704. https://doi.org/10.2337/diab.30.8.702

20. Schiaffino S, Reggiani C (2011) Fiber types in mammalian skeletal muscles. Physiol Rev 91(4):1447-1531. https://doi.org/10.1152/ physrev.00031.2010

21. Orchard TJ, Dorman JS, Maser RE et al (1990) Factors associated with avoidance of severe complications after $25 \mathrm{yr}$ of IDDM. Pittsburgh epidemiology of diabetes complications study I. Diabetes Care 13(7):741-747. https://doi.org/10.2337/diacare.13. 7.741

22. Orchard TJ, Dorman JS, Maser RE et al (1990) Prevalence of complications in IDDM by sex and duration: Pittsburgh epidemiology of diabetes complications study II. Diabetes 39(9):11161124. https://doi.org/10.2337/diab.39.9.1116

23. Costacou T, Edmundowicz D, Prince C, Conway B, Orchard TJ (2007) Progression of coronary artery calcium in type 1 diabetes mellitus. Am J Cardiol 100(10):1543-1547. https://doi.org/10. 1016/j.amjcard.2007.06.050

24. Criqui MH, Denenberg JO, Ix JH et al (2014) Calcium density of coronary artery plaque and risk of incident cardiovascular events. JAMA 311(3):271-278. https://doi.org/10.1001/jama.2013. 282535 
25. Goodpaster BH, Bertoldo A, Ng JM et al (2014) Interactions among glucose delivery, transport, and phosphorylation that underlie skeletal muscle insulin resistance in obesity and type 2 diabetes: studies with dynamic pet imaging. Diabetes 63(3):1058-1068. https://doi. org $/ 10.2337 / \mathrm{db} 13-1249$

26. Patlak CS, Blasberg RG (1985) Graphical evaluation of blood-tobrain transfer constants from multiple-time uptake data. Generalizations. J Cereb Blood Flow Metab 5(4):584-590. https:// doi.org/10.1038/jcbfm.1985.87

27. Rewers M, Zaccaro D, D'Agostino R et al (2004) Insulin sensitivity, insulinemia, and coronary artery disease: the Insulin Resistance Atherosclerosis Study. Diabetes Care 27(3):781-787. https://doi. org/10.2337/diacare.27.3.781

28. Orchard TJ, Olson JC, Erbey JR et al (2003) Insulin resistancerelated factors, but not glycemia, predict coronary artery disease in type 1 diabetes: 10-year follow-up data from the Pittsburgh epidemiology of diabetes complications study. Diabetes Care 26(5):1374-1379. https://doi.org/10.2337/diacare.26.5.1374

29. Cree-Green M, Newcomer BR, Brown MS et al (2015) Delayed skeletal muscle mitochondrial ADP recovery in youth with type 1 diabetes relates to muscle insulin resistance. Diabetes 64(2):383392. https://doi.org/10.2337/db14-0765

30. Kacerovsky M, Brehm A, Chmelik M et al (2011) Impaired insulin stimulation of muscular ATP production in patients with type 1 diabetes. J Intern Med 269(2):189-199. https://doi.org/10.1111/j. 1365-2796.2010.02298.x

31. Peltoniemi P, Yki-Järvinen H, Laine H et al (2001) Evidence for spatial heterogeneity in insulin- and exercise-induced increases in glucose uptake: studies in normal subjects and patients with type 1 diabetes. J Clin Endocrinol Metab 86(11):5525-5533. https://doi. org/10.1210/jcem.86.11.7979

32. Paternostro G, Camici PG, Lammerstma AA et al (1996) Cardiac and skeletal muscle insulin resistance in patients with coronary heart disease. A study with positron emission tomography. J Clin Invest 98(9):2094-2099. https://doi.org/10.1172/JCI119015
33. Nuutila P, Knuuti J, Ruotsalainen U et al (1993) Insulin resistance is localized to skeletal but not heart muscle in type 1 diabetes. Am J Phys 264(5 pt 1):E756-E762

34. Honka MJ, Latva-Rasku A, Bucci M et al (2018) Insulin-stimulated glucose uptake in skeletal muscle, adipose tissue and liver: a positron emission tomography study. Eur J Endocrinol 178(5):523531. https://doi.org/10.1530/EJE-17-0882

35. James DE, Zorzano A, Boni-Schnetzler M et al (1986) Intrinsic differences of insulin receptor kinase activity in red and white muscle. J Biol Chem 261(32):14939-14944

36. Gaster M, Staehr P, Beck-Nielsen H, Schrøder HD, Handberg A (2001) GLUT4 is reduced in slow muscle fibers of type 2 diabetic patients: is insulin resistance in type 2 diabetes a slow, type 1 fiber disease? Diabetes 50(6):1324-1329. https://doi.org/10.2337/ diabetes.50.6.1324

37. Coen PM, Dubé JJ, Amati F et al (2010) Insulin resistance is associated with higher intramyocellular triglycerides in type I but not type II myocytes concomitant with higher ceramide content. Diabetes 59(1):80-88. https://doi.org/10.2337/db09-0988

38. Talbot J, Maves L (2016) Skeletal muscle fiber type: using insights from muscle developmental biology to dissect targets for susceptibility and resistance to muscle disease. Wiley Interdiscip Rev Dev Biol 5:518-534. https://doi.org/10.1002/wdev.230

39. He J, Watkins S, Kelley DE (2001) Skeletal muscle lipid content and oxidative enzyme activity in relation to muscle fiber type in type 2 diabetes and obesity. Diabetes 50(4):817-823. https://doi. org/10.2337/diabetes.50.4.817

40. Gaster M, Poulsen P, Handberg A, Schrøder HD, Beck-Nielsen H (2000) Direct evidence of fiber type-dependent GLUT-4 expression in human skeletal muscle. Am J Physiol Endocrinol Metab 278(5): E910-E916. https://doi.org/10.1152/ajpendo.2000.278.5.e910

Publisher's note Springer Nature remains neutral with regard to jurisdictional claims in published maps and institutional affiliations. 